\title{
Strong convergence of an new iterative method for a zero of accretive operator and nonexpansive mapping
}

Meng Wen and Changsong $\mathrm{Hu}^{*}$

* Correspondence: huchang1004@yahoo.com.cn Department of Mathematics, Hubei Normal University, Huangshi 435002, P. R. China

\begin{abstract}
Let $E$ be a Banach space and $A$ an $m$-accretive operator with a zero. Consider the iterative method that generates the sequence $\left\{x_{n}\right\}$ by the algorithm $x_{n+1}=\alpha_{n} \gamma \phi\left(x_{n}\right)+\left(I-\alpha_{n} F\right) J_{r_{n}} x_{n}$, where $\left\{a_{n}\right\}$ and $\left\{r_{n}\right\}$ are two sequences satisfying certain conditions, $J_{r_{n}}$ denotes the resolvent $\left(I+r_{n} A\right)^{-1}$ for $r_{n}>0$, $F$ be a strongly positive bounded linear operator on $E$ is $0<\gamma<\bar{\gamma}$, and $\varphi$ be a MKC on E. Strong convergence of the algorithm $\left\{x_{n}\right\}$ is proved assuming $E$ either has a weakly continuous duality map or is uniformly smooth.
\end{abstract}

MSC: 47H09; 47H10

Keywords: MKC, accretive operators, the resolvent operator, iterative method, weakly continuous duality map

\section{Introduction}

Let $E$ be a real Banach space, $C$ a nonempty closed convex subset of $E$, and $T: C \rightarrow C$ a mapping. Recall that $T$ is nonexpansive if $\|T x-T y\| \leq\|x-y\|$ for all $x, y \in C$. A point $x \in C$ is a fixed point of $T$ provided $T x=x$. Denote by $F(T)$ the set of fixed points of $T$, that is, $F(T)=\{x \in C, T x=x\}$.

It is assumed throughout the paper that $T$ is a nonexpansive mapping such that $F(t) \neq \varnothing$. The normalized duality mapping $J$ from a Banach space $E$ into $2^{E^{*}}$ is given by $J(x)=\left\{f \in E^{*}:\langle x, f\rangle=\|x\|^{2}=\|f\|^{2}\right\}, x \in E$, where $E^{*}$ denotes the dual space of $E$ and $\langle.,$.$\rangle denotes the generalized duality pairing.$

Theorem 1.1. (Banach [1]). Let $(X, d)$ be a complete metric space and let $f$ be a contraction on $X$, that is, there exists $r \in(0,1)$ such that $d(f(x), f(y)) \leq r d(x, y)$ for all $x, y \in X$. Then $f$ has a unique fixed point.

Theorem 1.2. (Meir and Keeler [2]). Let $(X, d)$ be a complete metric space and let $\varphi$ be a Meir-Keeler contraction (MKC, for short) on $X$, that is, for every $\varepsilon>0$, there exists $\delta>0$ such that $\mathrm{d}(x, y)<\varepsilon+\delta$ implies $\mathrm{d}(\varphi(x), \varphi(y))<\varepsilon$ for all $x, y \in X$. Then $\varphi$ has a unique fixed point.

This theorem is one of generalizations of Theorem 1.1, because contractions are Meir-Keeler contractions.

Let $F$ be a strongly positive bounded linear operator on $E$, that is, there exists a constant $\tilde{\gamma}>0$ such that

(C) 2012 Wen and Hu; licensee Springer. This is an Open Access article distributed under the terms of the Creative Commons Attribution License (http://creativecommons.org/licenses/by/2.0), which permits unrestricted use, distribution, and reproduction in any medium, provided the original work is properly cited. 


$$
\langle F x, J(x)\rangle \geq \tilde{\gamma}\|x\|^{2},\|a I-b F\|=\sup _{\|x\| \leq 1}\{\mid\langle(a I-b F) x, J(x) \||: a \in[0,1], b \in[0,1]\},
$$

where $I$ is the identity mapping and $J$ is the normalized duality mapping.

Let $D$ be a subset of $C$. Then $Q: C \rightarrow D$ is called a retraction from $C$ onto $D$ if $Q(x)=x$ for all $x \in D$. A retraction $Q: C \rightarrow D$ is said to be sunny if $Q(x+t(x-Q(x)))=Q(x)$ for all $x \in C$ and $t \geq 0$ whenever $x+t(x-Q(x)) \in C$. A subset $D$ of $C$ is said to be a sunny nonexpansive retract of $C$ if there exists a sunny nonexpansive retraction of $C$ onto $D$. In a smooth Banach space $E$, it is known (cf. [[3], p. 48]) that $Q: C \rightarrow D$ is a sunny nonexpansive retraction if and only if the following condition holds:

$$
\langle x-Q(x), J(z-Q(x))\rangle \leq 0, \quad x \in C, z \in D .
$$

Recall that an operator $A$ with domain $D(A)$ and range $R(A)$ in $E$ is said to be accretive, if for each $x_{i} \in D(A)$ and $y_{i} \in A x_{i}, i=1,2$, there is a $j \in J\left(x_{2}-x_{1}\right)$ such that

$$
\left\langle y_{2}-y_{1}, j\right\rangle \geq 0 \text {. }
$$

An accretive operator $A$ is $\mathrm{m}$-accretive if $R(I+\lambda A)=E$ for all $\lambda>0$. Denote by $N(A)$ the zero set of $A$; i.e.,

$$
N(A):=A^{-1} 0=\{x \in D(A): A x=0\} .
$$

Throughout the rest of this paper it is always assumed that $A$ is m-accretive and $N$ $(A)$ is nonempty. Denote by $J_{r}$ the resolvent of $A$ for $r>0$ :

$$
J_{r}=(I+r A)^{-1} .
$$

Note that if $A$ is m-accretive, then $J_{r}: E \rightarrow E$ is nonexpansive and $F\left(J_{r}\right)=N(A)$ for all $r>0$. We also denote by $A_{r}$ the Yosida approximation of $A$, i.e., $A_{r}=\frac{1}{r}\left(I-J_{r}\right)$. It is well known that $J_{r}$ is a nonexpansive mapping from $E$ to $C:=D(A)$.

Recall that a gauge is a continuous strictly increasing function $\phi:[0, \infty) \rightarrow[0, \infty)$ such that $\phi(0)=0$ and $\phi(t) \rightarrow \infty$ as $t \rightarrow \infty$. Associated to a gauge $\phi$ is the duality mapping $J_{\phi}: E \rightarrow E^{*}$ defined by

$$
J_{\varphi}(x)=\left\{x^{*} \in E^{*}:\left\langle x, x^{*}\right\rangle=\|x\| \varphi(\|x\|),\left\|x^{*}\right\|=\varphi(\|x\|)\right\}, \quad x \in E .
$$

Following Browder [4], we say that a Banach space $E$ has a weakly continuous duality map if there exists a gauge $\phi$ for which the duality map $J_{\phi}$ is single-valued and weakto-weak* sequentially continuous(i.e., if $\left\{x_{n}\right\}$ is a sequence in $E$ weakly convergent to a point $x$, then the sequence $J_{\phi}\left(x_{n}\right)$ converges weakly* to $\left.J_{\phi}(x)\right)$. It is known that $l^{p}$ has a weakly continuous duality map for all $1<p<\infty$, with gauge $\phi(t)=t^{p-1}$. Set

$$
\Phi(t)=\int_{0}^{t} \varphi(\tau) d \tau, \quad t \geq 0 .
$$

Then

$$
J_{\varphi}(x)=\partial \Phi(\|x\|), \quad x \in E,
$$

where $\partial$ denotes the subdifferential in the sense of convex analysis. 
Recently, Hong-Kun Xu [5] introduced the following iterative scheme: for $x_{1}=x \in C$,

$$
x_{n+1}=\alpha_{n} u+\left(1+\alpha_{n}\right) J_{r_{n}} x_{n}, \quad \forall n \geq 1,
$$

where $\left\{a_{n}\right\}$ and $\left\{r_{n}\right\}$ are two sequences satisfying certain conditions, and $J_{r_{n}}$ denotes the resolvent $\left(I+r_{n} A\right)^{-1}$ for $r_{n}>0$. He proved the strong convergence of the algorithm $\left\{x_{n}\right\}$ assuming $E$ either has a weakly continuous duality map or is uniformly smooth.

Motivated and inspired by the results of Hong-Kun $\mathrm{Xu}$, we introduce the following iterative scheme: for any $x_{0} \in E$,

$$
x_{n+1}=\alpha_{n} \gamma \phi\left(x_{n}\right)+\left(I-\alpha_{n} F\right) J_{r_{n}} x_{n}, \quad \forall n \geq 0,
$$

where $\left\{a_{n}\right\}$ and $\left\{r_{n}\right\}$ are two sequences satisfying certain conditions, $J_{r_{n}}$ denotes the resolvent $\left(I+r_{n} A\right)^{-1}$ for $r_{n}>0, F$ be a strongly positive bounded linear operator on $E$ is $0<\gamma<\bar{\gamma}$, and $\varphi$ be a MKC on $E$. Strong convergence of the algorithm $\left\{x_{n}\right\}$ is proved assuming $E$ either has a weakly continuous duality map or is uniformly smooth. Our results extend and improve the corresponding results of Hong-Kun $\mathrm{Xu}$ [5] and many others.

\section{Preliminaries}

In order to prove our main results, we need the following lemmas.

Lemma 2.1. [5]. Assume that $E$ has a weakly continuous duality map $J_{\phi}$ with gauge $\phi$,

(i) For all $x, y \in E$, there holds the inequality

$$
\Phi(\|x+y\|) \leq \Phi(\|x\|)+\left\langle y, J_{\varphi}(x+y)\right\rangle .
$$

(ii) Assume a sequence $\left\{x_{n}\right\}$ in $E$ is weakly convergent to a point $x$, then there holds the equality

$$
\limsup _{n \rightarrow \infty} \Phi\left(\left\|x_{n}-y\right\|\right)=\limsup _{n \rightarrow \infty} \Phi\left(\left\|x_{n}-x\right\|\right)+\Phi(\|y-x\|), \quad x, y \in E .
$$

Lemma 2.2. [6,7]. Let $\left\{s_{n}\right\}$ be a sequence of nonnegative real numbers satisfying

$$
s_{n+1} \leq\left(1-\lambda_{n}\right) s_{n}+\lambda_{n} \delta_{n}+\gamma_{n}, \quad n \geq 0,
$$

where $\left\{\lambda_{n}\right\},\left\{\delta_{n}\right\}$ and $\left\{\gamma_{n}\right\}$ satisfy the following conditions:

(i) $\left\{\lambda_{n}\right\} \subset[0,1]$ and $\sum_{n=0}^{\infty} \lambda_{n}=\infty$,

(ii) $\lim \sup _{n \rightarrow \infty} \delta_{n} \leq 0$ or $\sum_{n=0}^{\infty} \lambda_{n} \delta_{n}<\infty$ (iii) $\gamma_{n} \geq 0(n \geq 0), \sum_{n=0}^{\infty} \gamma_{n}<\infty$. Then $\lim _{n \rightarrow \infty} s_{n}=0$.

Lemma 2.3. (The Resolvent Identity [8,9]). For $\lambda>0$ and $v>0$ and $x \in E$,

$$
J_{\lambda} x=J_{\nu}\left(\frac{v}{\lambda}+\left(1-\frac{v}{\lambda}\right) J_{\lambda} x\right) .
$$


Lemma 2.4. (see [ [10], Lemma 2.3]). Assume that $F$ is a strongly positive linear bounded operator on a smooth Banach space E with coefficient $\bar{\gamma}>0$ and $0<\rho \leq\|F\|^{-1}$. Then,

$$
\|I-\rho F\| \leq 1-\rho \bar{\gamma} .
$$

Lemma 2.5. (see [ [11], Lemma 2.3]). Let $\varphi$ be a MKC on a convex subset $C$ of a Banach space $E$. Then for each $\varepsilon>0$, there exists $r \in(0,1)$ such that

$$
\|x-y\| \geq \varepsilon \text { implies }\|\phi x-\phi y\| \leq r\|x-y\| \quad \forall x, y \in C .
$$

Lemma 2.6. Let $E$ be a reflexive Banach space which admits a weakly continuous duality map $J_{\phi}$ with gauge $\phi$. Let $T: E \rightarrow E$ be a nonexpansive mapping. Now given $\varphi$ : $E \rightarrow E$ be a $M K C, F$ be a strongly positive linear bounded operator with coefficient $\bar{\gamma}>0$. Assume that $0<\gamma<\bar{\gamma}$, the sequence $\left\{x_{t}\right\}$ defined by $x_{t}=t \gamma \varphi\left(x_{t}\right)+(I-t F) T x_{t}$. Then $T$ has a fixed point if and only if $\left\{x_{t}\right\}$ remains bounded as $t \rightarrow 0^{+}$, and in this case, $\left\{x_{t}\right\}$ converges as $t \rightarrow 0^{+}$strongly to a fixed point of $T$. If $\tilde{x}:=\lim _{t \rightarrow 0} x_{t}$, then $\tilde{x}$ uniquely solves the variational inequality

$$
\langle(F-\gamma \phi) \tilde{x}, J(\tilde{x}-p)\rangle \leq 0, \quad P \in F(T) .
$$

Proof. The definition of $\left\{x_{t}\right\}$ is well defined. Indeed, from the definition of MKC, we can see MKC is also a nonexpansive mapping. Consider a mapping $S_{t}$ on $E$ defined by

$$
S_{t}(x)=t \gamma \phi(x)+(I-t F) T x, \quad x \in E .
$$

It is easy to see that $S_{t}$ is a contraction. Indeed, by Lemma 2.4, we have

$$
\begin{aligned}
\left\|S_{t} x-S_{t} y\right\| & \leq t \gamma\|\phi(x)-\phi(\gamma)\|+\|(I-t F)(T x-T y)\| \\
& \leq t \gamma\|x-\gamma\|+(1-t \bar{\gamma})\|T x-T \gamma\| \\
& \leq[1-t(\bar{\gamma}-\gamma)]\|x-\gamma\|
\end{aligned}
$$

for all $x, y \in E$. Hence $S_{t}$ has a unique fixed point, denoted as $x_{t}$, which uniquely solves the fixed point equation

$$
x_{t}=t \gamma \phi\left(x_{t}\right)+(I-t F) T x_{t}, \quad x_{t} \in E .
$$

We next show the sequence $\left\{x_{t}\right\}$ is bounded. Indeed, we may assume $F(t) \neq \emptyset$ and with no loss of generality $t<\|F\|^{-1}$. Take $p \in F(T)$ to deduce that, for $t \in(0,1)$,

$$
\begin{aligned}
\left\|x_{t}-p\right\| & =\left\|t \gamma \phi\left(x_{t}\right)+(I-t F) T x_{t}-p\right\| \\
& =\left\|t\left(\gamma \phi\left(x_{t}\right)-F p\right)+(I-t F)\left(T x_{t}-p\right)\right\| \\
& \leq(1-t \bar{\gamma})\left\|x_{t}-p\right\|+t \gamma\left\|x_{t}-p\right\|+t\|\gamma \phi(p)-F p\| \\
& \leq[1-t(\bar{\gamma}-\gamma)]\left\|x_{t}-p\right\|+t\|\gamma \phi(p)-F p\| .
\end{aligned}
$$

Hence

$$
\left\|x_{t}-p\right\| \leq \frac{1}{\bar{\gamma}-\gamma}\|\gamma \phi(p)-F p\|
$$

and $\left\{x_{t}\right\}$ is bounded. 
Next assume that $\left\{x_{t}\right\}$ is bounded as $t \rightarrow 0^{+}$. Assume $t_{n} \rightarrow 0^{+}$and $\left\{x_{t_{n}}\right\}$ is bounded. Since $E$ is reflexive, we may assume that $x_{t_{n}} \rightarrow z$ for some $z \in E$. Since $J_{\phi}$ is weakly continuous, we have by Lemma 2.1,

$$
\limsup _{n \rightarrow \infty} \Phi\left(\left\|x_{t_{n}}-x\right\|\right)=\limsup _{n \rightarrow \infty} \Phi\left(\left\|x_{t_{n}}-z\right\|\right)+\Phi(\|x-z\|), \quad \forall x \in E .
$$

Put

$$
f(x)=\limsup _{n \rightarrow \infty} \Phi\left(\left\|x_{t_{n}}-x\right\|\right), \quad x \in E .
$$

It follows that

$$
f(x)=f(z)+\Phi(\|x-z\|), \quad x \in E .
$$

Since

$$
\left\|x_{t_{n}}-T x_{t_{n}}\right\|=t_{n}\left\|\gamma \phi\left(x_{t_{n}}\right)-F T x_{t_{n}}\right\| \rightarrow 0,
$$

we obtain

$$
\begin{aligned}
f(T z) & =\lim \sup _{n \rightarrow \infty} \Phi\left(\left\|x_{t_{n}}-T z\right\|\right)=\lim \sup _{n \rightarrow \infty} \Phi\left(\left\|T x_{t_{n}}-T z\right\|\right) \\
& \leq \lim \sup _{n \rightarrow \infty} \Phi\left(\left\|x_{t_{n}}-z\right\|\right)=f(z) .
\end{aligned}
$$

On the other hand, however,

$$
f(T z)=f(z)+\Phi(\|T z-z\|)
$$

Combining Equations (2.2) and (2.3) yields

$$
\Phi(\|T z-z\|) \leq 0 .
$$

Hence, $T z=z$ and $z \in F(T)$.

Finally, we prove that $\left\{x_{t}\right\}$ converges strongly to a fixed point of $T$ provided it remains bounded when $t \rightarrow 0$.

Let $\left\{t_{n}\right\}$ be a sequence in $(0,1)$ such that $t_{n} \rightarrow 0$ and $x_{t_{n}} \rightarrow z$ as $n \rightarrow \infty$. Then the argument above shows that $z \in F(T)$. We next show that $x_{t_{n}} \rightarrow z$. By contradiction, there is a number $\varepsilon_{0}>0$ such that $\left\|x_{t_{n}}-z\right\| \geq \varepsilon_{0}$. Then by Lemma 2.8, there is a number $r \in(0,1)$ such that

$$
\begin{aligned}
\left\|\phi\left(x_{t_{n}}\right)-\phi(z)\right\| & \leq r\left\|x_{t_{n}}-z\right\|, \\
\left\|x_{t_{n}}-z\right\| \varphi\left(\left\|x_{t_{n}}-z\right\|\right) & =\left\langle x_{t_{n}}-z_{1} J_{\varphi}\left(x_{t_{n}}-z\right)\right\rangle \\
& =\left\langle t_{n}\left(\gamma \phi\left(x_{t_{n}}\right)-F z\right)+\left(I-t_{n} F\right)\left(T x_{t_{n}}-z\right), J_{\varphi}\left(x_{t_{n}}-z\right)\right\rangle \\
& \leq t_{n}\left\langle\gamma \phi\left(x_{t_{n}}\right)-F z, J_{\varphi}\left(x_{n}-z\right)\right\rangle+\left\|\left(I-t_{n} F\right)\left(T x_{t_{n}}-z\right)\right\| \varphi\left(\left\|x_{t_{n}}-z\right\|\right) \\
& \leq\left(1-t_{n} \bar{\gamma}\right)\left\|x_{t_{n}}-z\right\| \varphi\left(\left\|x_{t_{n}}-z\right\|\right)+t_{n}\left\langle\gamma \phi\left(x_{t_{n}}\right)-F z, J_{\varphi}\left(x_{n}-z\right)\right\rangle .
\end{aligned}
$$

It follows that

$$
\begin{aligned}
\left\|x_{t_{n}}-z\right\| \varphi\left(\left\|x_{t_{n}}-z\right\|\right) & \leq \frac{1}{\bar{\gamma}}\left\langle\gamma \phi\left(x_{t_{n}}\right)-F z_{,} J_{\varphi}\left(x_{t_{n}}-z\right)\right\rangle \\
& =\frac{1}{\bar{\gamma}}\left[\left\langle\gamma \phi\left(x_{t_{n}}\right)-\gamma \phi(z), J_{\varphi}\left(x_{t_{n}}-z\right)\right\rangle+\left\langle\gamma \phi(z)-F z, J_{\varphi}\left(x_{t_{n}}-z\right)\right\rangle\right] \\
& \left.\leq \frac{\gamma}{\bar{\gamma}} r\left\|x_{t_{n}}-z\right\| \varphi\left(\left\|x_{t_{n}}-z\right\|\right)+\frac{1}{\bar{\gamma}}\left\langle\gamma \phi(z)-F z_{,} J_{\varphi}\left(x_{t_{n}}-z\right)\right\rangle\right] .
\end{aligned}
$$


Therefore,

$$
\left\|x_{t_{n}}-z\right\| \varphi\left(\left\|x_{t_{n}}-z\right\|\right) \leq \frac{1}{\bar{\gamma}-\gamma r}\left\langle\gamma \phi(z)-F z_{,} J_{\phi}\left(x_{t_{n}}-z\right)\right\rangle .
$$

Now observing that $x_{t_{n}} \rightarrow z$ implies $J_{\varphi}\left(x_{t_{n}}-z\right) \rightarrow 0$, we conclude from the last inequality that

$$
\lim _{n \rightarrow \infty}\left\|x_{t_{n}}-z\right\| \varphi\left(\left\|x_{t_{n}}-z\right\|\right)=0 .
$$

It contradicts $\left\|x_{t_{n}}-z\right\| \varphi\left(\left\|x_{t_{n}}-z\right\|\right) \geq \varepsilon_{0} \varphi\left(\varepsilon_{0}\right)>0$. Hence $x_{t_{n}} \rightarrow z$.

We finally prove that the entire net $\left\{x_{t}\right\}$ converges strongly. Towards this end, we assume that two null sequences $\left\{t_{n}\right\}$ and $\left\{s_{n}\right\}$ in $(0,1)$ are such that

$$
t_{n} \rightarrow 0, x_{t_{n}} \rightarrow z \text { and } s_{n} \rightarrow 0, \quad x_{s_{n}} \rightarrow z
$$

We have to show $z=z$. Indeed, for $p \in F(T)$. Since

$$
x_{t}=t \gamma \phi\left(x_{t}\right)+(I-t f) T x_{t}
$$

we derive that

$$
(F-\gamma \phi) x_{t}=-\frac{1}{t}(I-t F)(I-T) x_{t}
$$

Notice

$$
\begin{aligned}
\left\langle(I-T) x_{t}-(I-T) p, J_{\varphi}\left(x_{t}-p\right)\right\rangle & =\left\|x_{t}-p\right\| \varphi\left(\left\|x_{t}-p\right\|\right)+\left\langle T p-T x_{t}, J_{\varphi}\left(x_{t}-p\right)\right\rangle \\
& \geq\left\|x_{t}-p\right\| \varphi\left(\left\|x_{t}-p\right\|\right)-\left\|T p-T x_{t}\right\|\left\|J_{\varphi}\left(x_{t}-p\right)\right\| \\
& \geq\left\|x_{t}-p\right\|\left[\varphi\left(\left\|x_{t}-p\right\|\right)-\varphi\left(\left\|x_{t}-p\right\|\right)\right] \\
& =0 .
\end{aligned}
$$

It follows that,

$$
\begin{aligned}
\left\langle(F-\gamma \phi) x_{t}, J_{\varphi}\left(x_{t}-p\right)\right\rangle & =-\frac{1}{t}\left\langle(I-t F)(I-T) x_{t} J_{\varphi}\left(x_{t}-p\right)\right\rangle \\
& \leq\left\langle\left(F(I-T) x_{t} J_{\varphi}\left(x_{t}-p\right)\right\rangle .\right.
\end{aligned}
$$

Now replacing $t$ in (2.5) with $t_{n}$ and letting $n \rightarrow \infty$, noticing $(I-T) x_{t_{n}} \rightarrow(I-T) z=0$ for $z \in F(T)$, we obtain $\left\langle(F-\gamma \varphi) z, J_{\phi}(z-p)\right\rangle \leq 0$. In the same way, we have $\left\langle(F-\gamma \phi) z_{1} J_{\varphi}(\dot{z}-p)\right\rangle \leq 0$.

Thus, we have

$$
\left\langle(F-\gamma \phi) z_{1} J_{\varphi}(z-\dot{z})\right\rangle \leq 0 \quad \text { and } \quad\left\langle(F-\gamma \phi) \dot{z}_{1} J_{\varphi}(z-z)\right\rangle \leq 0 .
$$

Adding up (2.6) gets

$$
\left\langle(F-\gamma \phi) z-(F-\gamma \phi) \dot{z}, \quad J_{\varphi}(z-\dot{z})\right\rangle \leq 0 .
$$

On the other hand, without loss of generality, we may assume there is a number $\varepsilon$ such that $\|z-z\| \geq \varepsilon$, then by Lemma 2.5 there is a number $r_{1}$ such that $\|\phi(z)-\phi(z)\| \leq r_{1}\|z-\dot{z}\|$. Noticing that 


$$
\begin{aligned}
J_{\varphi} & =(\varphi(\|x\|) /\|x\|) J(x) \quad x \neq 0, \\
\left\langle(F-\gamma \phi) z-(F-\gamma \phi) \dot{z}_{,} J_{\varphi}(z-\dot{z})\right\rangle & =\left\langle F(z-\dot{z}), J_{\varphi}(z-\dot{z})\right\rangle-\left\langle\gamma \phi z-\gamma \phi \dot{z}, J_{\varphi}(z-\dot{z})\right\rangle \\
& \geq \bar{\gamma}\|z-\dot{z}\|\left\|J_{\varphi}(z-\dot{z})\right\|-\gamma r_{1}\|z-\dot{z}\|\left\|J_{\varphi}(z-\dot{z})\right\| \\
& \geq\left(\bar{\gamma}-\gamma r_{1}\right)\|z-\dot{z}\| \varphi(\|z-\dot{z}\|) \\
& >0 .
\end{aligned}
$$

Hence $z=\dot{z}$ and $\left\{x_{t}\right\}$ converges strongly. Thus we may assume $x_{t} \rightarrow \tilde{x}$. Since we have proved that, for all $t \in(0,1)$ and $p \in F(T)$,

$$
\left\langle(F-\gamma \phi) x_{t}, J_{\varphi}\left(x_{t}-p\right)\right\rangle \leq\left\langle\left(F(I-T) x_{t}, J_{\varphi}\left(x_{t}-p\right)\right\rangle,\right.
$$

letting $t \rightarrow 0$, we obtain that

$$
\left\langle(F-\gamma \phi) \tilde{x}, J_{\varphi}(\tilde{x}-p)\right\rangle \leq 0 .
$$

This implies that

$$
\langle(F-\gamma \phi) \tilde{x}, J(\tilde{x}-p)\rangle \leq 0 .
$$

Lemma 2.7. (see [12]). Assume that $C_{2} \geq C_{1}>0$. Then $\left\|J_{C_{1}} x-x\right\| \leq 2\left\|J_{C_{2}} x-x\right\|$ for all $x \in E$.

Lemma 2.8. [13]. Let $C$ be a nonempty closed convex subset of a reflexive Banach space $E$ which satisfies Opial's condition, and suppose $T: C \rightarrow E$ is a nonexpansive mapping. Then the mapping $I-T$ is demiclosed at zero, that is $x_{n} \rightarrow x$ and $\left\|x_{n}-T x_{n}\right\|$ $\rightarrow 0$, then $x=T x$.

Lemma 2.9. In a smooth Banach space E there holds the inequality

$$
\|x+y\|^{2} \leq\|x\|^{2}+2\langle y, J(x+y)\rangle, \quad x, y \in E .
$$

\section{Main result}

Theorem 3.1. Suppose that $E$ is reflexive which admits a weakly continuous duality map $J_{\phi}$ with gauge $\phi$ and $A$ is an m-accretive operator in $E$ such that $F^{*}=N(A) \neq \emptyset$. Now given $\varphi: E \rightarrow E$ be a MKC, and let $F$ be a strongly positive linear bounded operator on $E$ with coefficient $\bar{\gamma}>0,0<\gamma<\bar{\gamma}$. Assume

(i) $\lim _{n \rightarrow \infty} \alpha_{n}=0, \sum_{n=0}^{\infty} \alpha_{n}=\infty$;

(ii) $r_{n} \rightarrow \infty$.

Then $\left\{x_{n}\right\}$ defined by (1.4) converges strongly to a point in $F^{*}$.

Proof. First notice that $\left\{x_{n}\right\}$ is bounded. Indeed, take $p \in F^{*}$ to get

$$
\begin{aligned}
\left\|x_{n+1}-p\right\| & =\left\|\alpha_{n} \phi\left(x_{n}\right)+\left(I-\alpha_{n} F\right) J_{r_{n}} x_{n}-p\right\| \\
& =\left\|\alpha_{n} \gamma \phi\left(x_{n}\right)-\alpha_{n} F p+\alpha_{n} F p+\left(I-\alpha_{n} F\right) J_{r_{n}} x_{n}-p\right\| \\
& =\left\|\alpha_{n}\left(\gamma \phi\left(x_{n}\right)-F p\right)+\left(I-\alpha_{n} F\right)\left(J_{r_{n}} x_{n}-p\right)\right\| \\
& \leq\left(1-\alpha_{n} \bar{\gamma}\right)\left\|x_{n}-p\right\|+\alpha_{n} \gamma\left\|x_{n}-p\right\|+\alpha_{n}\|\gamma \phi(p)-F p\| \\
& \leq\left[1-\alpha_{n}(\bar{\gamma}-\gamma)\right]\left\|x_{n}-p\right\|+\alpha_{n}\|\gamma \phi(p)-F p\| \\
& \leq\left[1-\alpha_{n}(\bar{\gamma}-\gamma)\right]\left\|x_{n}-p\right\|+\alpha_{n}(\bar{\gamma}-\gamma) \frac{\|\gamma \phi(p)-F p\|}{\bar{\gamma}-\gamma} \\
& \leq \max \left\{\left\|x_{n}-p\right\|, \frac{\|\gamma \phi(p)-F p\|}{\bar{\gamma}-\gamma}\right\}, \quad n \geq 0 .
\end{aligned}
$$


By induction, we have

$$
\left\|x_{n}-p\right\| \leq \max \left\{\left\|x_{0}-p\right\|, \frac{\|\gamma \phi(p)-F p\|}{\bar{\gamma}-\gamma}\right\}, \quad n \geq 0 .
$$

This implies that $\left\{x_{n}\right\}$ is bounded and hence

$$
\begin{aligned}
\left\|x_{n+1}-J_{r_{n}} x_{n}\right\| & =\left\|\alpha_{n} \gamma \phi\left(x_{n}\right)+\left(I-\alpha_{n} F\right) J_{I_{n}} x_{n}-J_{r_{n}} x_{n}\right\| \\
& =\alpha_{n}\left\|\gamma \phi\left(x_{n}\right)-F J_{r_{n}} x_{n}\right\| \rightarrow 0 .
\end{aligned}
$$

We next prove that

$\lim \sup _{n \rightarrow \infty}\left\langle\gamma \varphi(p)-F p, J_{\phi}\left(x_{n}-p\right)\right\rangle \leq 0$, where $p=\lim _{t \rightarrow 0} x_{t}$ with $x_{t}=t \gamma \phi\left(x_{t}\right)+(I-t F) J_{r_{n}} x_{t}$.

Since $\left\{x_{n}\right\}$ is bounded, take a subsequence $\left\{x_{n_{k}}\right\}$ of $\left\{x_{n}\right\}$ such that

$$
\limsup _{n \rightarrow \infty}\left\langle\gamma \phi(p)-F p, J_{\phi}\left(x_{n}-p\right)\right\rangle=\lim _{k \rightarrow \infty}\left\langle\gamma \phi(p)-F p, J_{\varphi}\left(x_{n_{k}}-p\right)\right\rangle .
$$

Since $E$ is reflexive, we may further assume that $x_{n_{k}} \rightarrow \tilde{x}$. Moreover, since

$$
\left\|x_{n+1}-J_{r_{n}} x_{n}\right\| \rightarrow 0,
$$

we obtain

$$
J_{n_{n_{k}}-1} x_{n_{k}}-1-\tilde{x} .
$$

Taking the limit as $k \rightarrow \infty$ in the relation

$$
\left[J_{r_{n_{k}}-1} x_{n_{k}-1}, A_{r_{n_{k}}-1} x_{n_{k}}-1\right] \in A \text {, }
$$

we get $[\tilde{x}, 0] \in A$. That is, $\tilde{x} \in F^{*}$. Hence by (3.1) and Lemma 2.6 we have

$$
\limsup _{n \rightarrow \infty}\left\langle\gamma \phi(p)-F p, J_{\varphi}\left(x_{n}-p\right)\right\rangle=\lim _{k \rightarrow \infty}\left\langle\gamma \phi(p)-F p, J_{\varphi}\left(x_{n_{k}}-p\right)\right\rangle=\left\langle\gamma \phi(p)-F p, J_{\varphi}(\tilde{x}-p)\right\rangle \leq 0 .
$$

Finally to prove that $x_{n} \rightarrow p$, we apply Lemma 2.1 to get

$$
\begin{aligned}
\Phi\left(\left\|x_{n+1}-p\right\|\right) & \left.=\Phi\left(\| \alpha_{n} \gamma \phi\left(x_{n}\right)+\left(I-\alpha_{n} F\right)\right)_{r_{n}} x_{n}-p \|\right) \\
& =\Phi\left(\left\|\left(I-\alpha_{n} F\right)\left(J_{r_{n}} x_{n}-p\right)+\alpha_{n}\left(\gamma \phi\left(x_{n}\right)-F p\right)\right\|\right) \\
& =\Phi\left(\left\|\left(I-\alpha_{n} F\right)\left(J_{r_{n}} x_{n}-p\right)+\alpha_{n}\left(\gamma \phi\left(x_{n}\right)-\gamma \phi(p)\right)+\alpha_{n}(\gamma \phi(p)-F p)\right\|\right) \\
& \left.\leq \Phi\left(\left\|\left(I-\alpha_{n} F\right)\left(J_{r_{n}} x_{n}-p\right)+\alpha_{n}\left(\gamma \phi\left(x_{n}\right)-\gamma \phi(p)\right)\right\|\right)\right)+\alpha_{n}\left\langle\gamma \phi(p)-F p_{r} J_{\varphi}\left(x_{n+1}-p\right)\right\rangle \\
& \left.\leq\left[1-\alpha_{n}(\bar{\gamma}-\gamma)\right] \Phi\left(\left\|x_{n}-p\right\|\right)+\alpha_{n}\left\{\gamma \phi(p)-F p_{,}\right)_{\varphi}\left(x_{n+1}-p\right)\right\rangle .
\end{aligned}
$$

An application of Lemma 2.2 yields that $\Phi\left(\left\|x_{n}-p\right\|\right) \rightarrow 0$. That is, $\left\|x_{n}-p\right\| \rightarrow 0$, i.e., $x_{n} \rightarrow p$. The proof is complete.

Theorem 3.2. Suppose that $E$ is reflexive which admits a weakly continuous duality map $J_{\phi}$ with gauge $\phi$ and $A$ is an m-accretive operator in $E$ such that $F^{*}=N(A) \neq \emptyset$. Now given $\varphi: E \rightarrow E$ be a $M K C$, and let $F$ be a strongly positive linear bounded operator on $E$ with coefficient $\bar{\gamma}>0,0<\gamma<\bar{\gamma}$. Assume

(i) $\lim _{n \rightarrow \infty} \alpha_{n}=0, \sum_{n=0}^{\infty} \alpha_{n}=\infty$, and $\sum_{n=1}^{\infty}\left|\alpha_{n+1}-\alpha_{n}\right|<\infty\left(\right.$ e.g., $\left.\alpha_{n}=\frac{1}{n}\right)$;

(ii) $r_{n} \geq \varepsilon$ for all $n$ and $\sum_{n=1}^{\infty}\left|r_{n+1}-r_{n}\right|<\infty\left(\right.$ e.g., $\left.r_{n}=1+\frac{1}{n}\right)$. 
Then $\left\{x_{n}\right\}$ defined by (1.4) converges strongly to a point in $F^{*}$.

Proof. We only include the differences. We have

$$
x_{n+1}=\alpha_{n} \gamma \phi\left(x_{n}\right)+\left(I-\alpha_{n} F\right) J_{r_{n}} x_{n}, \quad x_{n}=\alpha_{n-1} \gamma \phi\left(x_{n-1}\right)+\left(I-\alpha_{n-1} F\right) J_{r_{n-1}} x_{n-1} .
$$

Thus,

$$
x_{n+1}-x_{n}=\left(I-\alpha_{n} F\right)\left(J_{r_{n}} x_{n}-J_{r_{n-1}} x_{n-1}\right)+\alpha_{n} \gamma \phi\left(x_{n}\right)+\alpha_{n-1} \gamma \phi\left(x_{n-1}\right)+\left(\alpha_{n}-\alpha_{n-1}\right) F J_{r_{n-1}} x_{n-1} .
$$

If $r_{n-1} \leq r_{n}$, using the resolvent identity

$$
J_{r_{n}} x_{n}=J_{r_{n-1}}\left(\frac{r_{n-1}}{r_{n}} x_{n}+\left(1-\frac{r_{n-1}}{r_{n}}\right) J_{r_{n}} x_{n}\right),
$$

we obtain

$$
\begin{aligned}
\left\|J_{r_{n}} x_{n}-J_{r_{n-1}} x_{n-1}\right\| & \leq \frac{r_{n-1}}{r_{n}}\left\|x_{n}-x_{n-1}\right\|+\left(1-\frac{r_{n-1}}{r_{n}}\right)\left\|J_{r_{n}} x_{n}-x_{n-1}\right\| \\
& \leq\left\|x_{n}-x_{n-1}\right\|+\left(\frac{r_{n}-r_{n-1}}{r_{n}}\right)\left\|J_{r_{n}} x_{n}-x_{n-1}\right\| \\
& \leq\left\|x_{n}-x_{n-1}\right\|+\frac{1}{\varepsilon}\left|r_{n-1}-r_{n}\right|\left\|J_{r_{n}} x_{n}-x_{n-1}\right\|
\end{aligned}
$$

It follows from (3.2) that

$$
\left\|x_{n+1}-x_{n}\right\| \leq\left(1-\alpha_{n}(\bar{\gamma}-\gamma)\right)\left\|x_{n}-x_{n-1}\right\|+M\left(\left|\alpha_{n}-\alpha_{n-1}\right|+\left|r_{n-1}-r_{n}\right|\right),
$$

where $M>0$ is some appropriate constant. Similarly we can prove (3.3) if $r_{n-1} \geq r_{n}$. By assumptions (i) and (ii) and Lemma 2.2, we conclude that

$$
\left\|x_{n+1}-x_{n}\right\| \rightarrow 0
$$

This implies that

$$
\left\|x_{n}-J_{r_{n}} x_{n}\right\| \leq\left\|x_{n+1}-x_{n}\right\|+\left\|x_{n+1}-J_{r_{n}} x_{n}\right\| \rightarrow 0,
$$

since $\left\|x_{n+1}-J_{r_{n}} x_{n}\right\|=\alpha_{n}\left\|\gamma \phi\left(x_{n}\right)-F J_{r_{n}} x_{n}\right\| \rightarrow 0$. It follows that

$$
\left\|A_{r_{n}} x_{n}\right\|=\frac{1}{r_{n}}\left\|x_{n}-J_{r_{n}} x_{n}\right\| \leq \frac{1}{\varepsilon}\left\|x_{n}-J_{r_{n}} x_{n}\right\| \rightarrow 0 .
$$

Now if $\left\{x_{n_{k}}\right\}$ is a subsequence of $\left\{x_{n}\right\}$ converging weakly to a point $\tilde{x}$, then taking the limit as $k \rightarrow \infty$ in the relation

$$
\left[J_{r_{n_{k}}} x_{n_{k}}, A_{r_{n_{k}}} x_{n_{k}}\right] \in A,
$$

we get $[\tilde{x}, 0] \in A$; i.e., $\tilde{x} \in F^{*}$. We therefore conclude that all weak limit points of $\left\{x_{n}\right\}$ are zeros of $A$.

The rest of the proof follows that of Theorem 3.1.

Finally, we consider the framework of uniformly smooth Banach spaces. Assume $r_{n} \geq$ $\varepsilon$ for some $\varepsilon>0$ (not necessarily $r_{n} \rightarrow \infty$ ), A is an m-accretive operator in $E$. Moreover let $\varphi: E \rightarrow E$ be a MKC and $F$ be a strongly positive linear bounded operator on $E$. Since $J_{r_{n}}$ is nonexpansive, the map $S: x \in E \mapsto t \gamma \phi(x)+(I-t F) J_{r_{n}} x$ is a contraction and for each integer $n \geq 1$ it has a unique fixed $z_{t, n} \in E$. Hence the scheme

$$
z_{t, n}=t \gamma \phi\left(z_{t, n}\right)+(I-t F) J_{r_{n}} z_{t, n}
$$


is well defined.

Note that $\left\{z_{t, n}\right\}$ is uniformly bounded; indeed, $\left\|z_{t, n}-p\right\| \leq \frac{1}{\bar{\gamma}-\gamma}\|\gamma \phi(p)-F p\|$ for all $t \in(0,1), n \geq 1$ and $p \in F^{*}$. A key component of the proof of the next theorem is the following lemma.

Lemma 3.1. The limit $\hat{z}=\lim _{t \rightarrow 0} z_{t, n}$ is uniform for all $n \geq 1$.

Proof. It suffices to show that for any positive integer $n_{t}$ (which may depend on $t \in$ $(0,1))$, if $z_{t, n_{t}} \in E$ is the unique point in $E$ that satisfies the property

$$
z_{t, n_{t}}=t \gamma \phi\left(z_{t, n_{t}}\right)+(I-t F) J_{r_{n_{t}}} z_{t, n_{t}}
$$

then $\left\{z_{t, n_{t}}\right\}$ converges as $t \rightarrow 0$ to a point in $F^{*}$. For simplicity put

$$
w_{t}=z_{t, n_{t}} \quad \text { and } \quad V_{t}=J_{r_{n_{t}}} .
$$

It follows that

$$
w_{t}=t \gamma \phi\left(w_{t}\right)+(I-t F) V_{t} w_{t} .
$$

Note that $\operatorname{Fix}\left(V_{t}\right)=F^{*}$ for all $t$. Note also that $\left\{w_{t}\right\}$ is bounded; indeed, we have $\left\|w_{t}-p\right\| \leq \frac{1}{\bar{\gamma}-\gamma}\|\gamma \phi(p)-F p\|$ for all $t \in(0,1)$ and $p \in F^{*}$. Since $\left\{V_{t} w_{t}\right\}$ is bounded, it is easy to see that

$$
\left\|w_{t}-V_{t} w_{t}\right\|=t\left\|\gamma \phi\left(w_{t}\right)-F V_{t} w_{t}\right\| \rightarrow 0, \quad \text { as } \quad t \rightarrow 0 .
$$

Since $r_{n} \geq \varepsilon$ for all $n$, by Lemma 2.7 , we have

$$
\left\|w_{t}-J_{\varepsilon} w_{t}\right\| \leq 2\left\|w_{t}-J_{r_{t}} w_{t}\right\|=2\left\|w_{t}-V_{t} w_{t}\right\| \rightarrow 0 .
$$

Let $\left\{t_{k}\right\}$ be a sequence in $(0,1)$ such that $t_{k} \rightarrow 0$ as $k \rightarrow \infty$. Define a function $f$ on $E$ by

$$
f(w)=\operatorname{LIM}_{k} \frac{1}{2}\left\|w_{t_{k}}-w\right\|^{2}, \quad w \in E
$$

where LIM denotes a Banach limit on $l^{\infty}$. Let

$$
K:=\{w \in E: f(w)=\min \{f(y): y \in E\}\} .
$$

Then $K$ is a nonempty closed convex bounded subset of $E$. We claim that $K$ is also invariant under the nonexpansive mapping $J_{\varepsilon}$. Indeed, noting (3.8), we have for $w \in K$,

$$
\begin{aligned}
f\left(J_{\varepsilon} w\right) & =\operatorname{LIM}_{k} \frac{1}{2}\left\|w_{t_{k}}-J_{\varepsilon} w\right\|^{2}=\operatorname{LIM}_{k} \frac{1}{2}\left\|J_{\varepsilon} w_{t_{k}}-J_{\varepsilon} w\right\|^{2} \\
& \leq \operatorname{LIM}_{k} \frac{1}{2}\left\|w_{t_{k}}-w\right\|^{2}=f(w) .
\end{aligned}
$$

Since a uniformly smooth Banach space has the fixed point property for nonexpansive mappings and since $J_{\varepsilon}$ is a nonexpansive self-mapping of $E$, $J_{\varepsilon}$ has a fixed point in $K$, say $w^{\prime}$. Now since $w^{\prime}$ is also a minimizer of $f$ over $E$, it follows that, for $w \in E$,

$$
\begin{aligned}
0 & \leq \frac{f\left(w^{\prime}+\lambda\left(w-w^{\prime}\right)\right)-f\left(w^{\prime}\right)}{\lambda} \\
& =\operatorname{LIM}_{k} \frac{\frac{1}{2}\left\|w_{t_{k}}-w^{\prime}+\lambda\left(w^{\prime}-w\right)\right\|^{2}-\frac{1}{2}\left\|w_{t_{k}}-w^{\prime}\right\|^{2}}{\lambda} .
\end{aligned}
$$


Since $E$ is uniformly smooth, the duality map $J$ is uniformly continuous on bounded sets, letting $\lambda \rightarrow 0^{+}$in the last equation yields

$$
0 \leq \operatorname{LIM}_{k}\left\langle w^{\prime}-w_{1} J\left(w_{t_{k}}-w^{\prime}\right)\right\rangle, \quad w \in E .
$$

Since

$$
w_{t_{k}}-w^{\prime}=\left(I-t_{k} F\right)\left(V_{t_{k}} w_{t_{k}}-w^{\prime}\right)+t_{k}\left(\gamma \phi\left(w_{t_{k}}\right)-F w^{\prime}\right),
$$

we obtain

$$
\begin{aligned}
\left\|w_{t_{n}}-w^{\prime}\right\|^{2} & =t_{k}\left\langle\gamma \phi\left(w_{t_{k}}\right)-F w^{\prime}, J\left(w_{t_{k}}-w^{\prime}\right)\right\rangle+\left\langle\left(I-t_{k} F\right)\left(V_{t_{k}} w_{t_{k}}-w^{\prime}\right), J\left(w_{t_{k}}-w^{\prime}\right)\right\rangle \\
& \leq t_{k}\left\langle\gamma \phi\left(w_{t_{k}}\right)-F w^{\prime}, J\left(w_{t_{k}}-w^{\prime}\right)\right\rangle+\left(1-t_{k} \bar{\gamma}\right)\left\|w_{t_{t_{k}}}-w^{\prime}\right\|^{2} \\
& \leq t_{k}\left(\gamma \phi\left(w_{t_{k}}\right)-\gamma \phi\left(w^{\prime}\right), J\left(w_{t_{k}}-w^{\prime}\right)\right\rangle+t_{k}\left\langle\gamma \phi\left(w^{\prime}\right)-F w^{\prime}, J\left(w_{t_{k}}-w^{\prime}\right)\right\rangle+\left(1+t_{k} \bar{\gamma}\right)\left\|w_{t_{k}}-w^{\prime}\right\|^{2} \\
& \leq\left[1-t_{k}(\bar{\gamma}-\gamma)\right]\left\|w_{t_{k}}-w^{\prime}\right\|^{2}+t_{k}\left\langle\gamma \phi\left(w^{\prime}\right)-F w^{\prime}, J\left(w_{t_{k}}-w^{\prime}\right)\right\rangle .
\end{aligned}
$$

It follows that

$$
\left\|w_{t_{k}}-w^{\prime}\right\|^{2} \leq \frac{1}{\bar{\gamma}-\gamma}\left\langle\gamma \phi\left(w^{\prime}\right)-F w^{\prime}, J\left(w_{t_{k}}-w^{\prime}\right)\right\rangle .
$$

Upon letting $w=\gamma \varphi\left(w^{\prime}\right)-F w^{\prime}+w^{\prime}$ in (3.9), we see that the last equation implies

$$
\operatorname{LIM}_{k}\left\|w_{t_{k}}-w^{\prime}\right\|^{2} \leq 0 .
$$

Therefore, $\left\{w_{t_{k}}\right\}$ contains a subsequence, still denoted $\left\{w_{t_{k}}\right\}$, converging strongly to $w_{1}$ (say). By virtue of (3.8), $w_{1}$ is a fixed point of $J_{\xi}$; i.e., a point in $F^{*}$.

To prove that the entire net $\left\{w_{t}\right\}$ converges strongly, assume $\left\{s_{k}\right\}$ is another null subsequence in $(0,1)$ such that $w_{s_{k}} \rightarrow w_{2}$ strongly. Then $w_{2} \in F^{*}$.

Repeating the argument of (3.10) we obtain

$$
\left\|w_{t}-w^{\prime}\right\|^{2} \leq \frac{1}{\bar{\gamma}-\gamma}\left\langle\gamma \phi\left(w^{\prime}\right)-F w^{\prime}, J\left(w_{t}-w^{\prime}\right)\right\rangle, \quad \forall w^{\prime} \in F^{*} .
$$

In particular,

$$
\left\|w_{1}-w_{2}\right\|^{2} \leq \frac{1}{\bar{\gamma}-\gamma}\left\langle\gamma \phi\left(w_{1}\right)-F w_{1}, J\left(w_{2}-w_{1}\right)\right\rangle
$$

and

$$
\left\|w_{2}-w_{1}\right\|^{2} \leq \frac{1}{\bar{\gamma}-\gamma}\left\langle\gamma \phi\left(w_{2}\right)-F w_{2}, J\left(w_{1}-w_{2}\right)\right\rangle .
$$

Adding up the last two equations gives

$$
\left\|w_{1}-w_{2}\right\|^{2} \leq 0 .
$$

That is, $w_{1}=w_{2}$. This concludes the proof.

Theorem 3.3. Suppose that $E$ is a uniformly smooth Banach space and $A$ is an $m$ accretive operator in $E$ such that $F^{*}=N(A) \neq \emptyset$. Now given $\varphi: E \rightarrow E$ be a $M K C$, and let $F$ be a strongly positive linear bounded operator on $E$ with coefficient $\bar{\gamma}>0,0<\gamma<\bar{\gamma}$. Assume 
(i) $\lim _{n \rightarrow \infty} \alpha_{n}=0, \sum_{n=0}^{\infty} \alpha_{n}=\infty$, and $\sum_{n=1}^{\infty}\left|\alpha_{n+1}-\alpha_{n}\right|<\infty\left(e . g ., \alpha_{n}=\frac{1}{n}\right)$;

(ii) $\lim _{n \rightarrow \infty}=r_{n}=r, r \in R^{+}, r_{n} \geq \varepsilon$ for all $n$ and $\sum_{n=1}^{\infty}\left|r_{n+1}-r_{n}\right|<\infty\left(\right.$ e.g., $\left.r_{n}=1+\frac{1}{n}\right)$.

Then $\left\{x_{n}\right\}$ defined by (1.4) converges strongly to a point in $F^{*}$.

Proof. Since

$$
\begin{aligned}
\left\|J_{r_{n}} x_{n}-J_{r} x_{n}\right\| & \leq\left\|J_{r}\left(\frac{r}{r_{n}} x_{n}+\left(1-\frac{r}{r_{n}}\right) J_{r_{n}} x_{n}\right)-J_{r} x_{n}\right\| \\
& \leq\left\|\left(\frac{r}{r_{n}} x_{n}+\left(1-\frac{r}{r_{n}}\right) J_{r_{n}} x_{n}\right)-x_{n}\right\| \\
& \leq\left|1-\frac{r}{r_{n}}\right|\left\|J_{r_{n}} x_{n}-x_{n}\right\| \rightarrow 0 \text { as }(n \rightarrow \infty) .
\end{aligned}
$$

Thus

$$
\left\|x_{n}-J_{r} x_{n}\right\| \leq\left\|x_{n}-J_{r_{n}} x_{n}\right\|+\left\|J_{r_{n}} x_{n}-J_{r} x_{n}\right\| \rightarrow 0 .
$$

We next claim that $\lim \sup _{n \rightarrow \infty}\left\langle\gamma \phi(\hat{z})-F \hat{z}, J\left(x_{n}-\hat{z}\right)\right\rangle \leq 0$, where $\hat{z}=\lim _{t \rightarrow 0} z_{t, n}$ with $z_{t, n}=t \gamma \varphi\left(z_{t, n}\right)+(I-t F) J_{r} z_{t, n}$.

For this purpose, let $\left\{x_{n_{k}}\right\}$ be a subsequence chosen in such a way that $\lim \sup _{n \rightarrow \infty}\left\langle\gamma \phi(\hat{z})-F \hat{z}, J\left(x_{n}-\hat{z}\right)\right\rangle=\lim _{k \rightarrow \infty}\left\langle\gamma \phi(\hat{z})-F \hat{z}, J\left(x_{n_{k}}-\hat{z}\right)\right\rangle \quad$ and $x_{n_{k}}-\tilde{x}$. Moreover, since $\left\|x_{n}-J_{r} x_{n}\right\| \rightarrow 0$, using Lemma 2.8, we know $\tilde{x} \in F\left(J_{r}\right)$. Hence by Lemma 2.6, we have

$$
\left.\limsup _{n \rightarrow \infty}\left\langle\gamma \phi(\hat{z})-F \hat{z}, J\left(x_{n}-\hat{z}\right)\right\rangle=\lim _{k \rightarrow \infty}\left\langle\gamma \phi(\hat{z})-F \hat{z}_{,} J\left(x_{n_{k}}-\hat{z}\right)\right\rangle=\langle\gamma \phi(\hat{z})-F \hat{z}, \tilde{x}-\hat{z})\right\rangle \leq 0 .
$$

Finally to prove that $x_{n} \rightarrow \hat{z}$ strongly, we write

$$
x_{n+1}-\hat{z}=\left(I-\alpha_{n} F\right)\left(J_{r_{n}} x_{n}-\hat{z}\right)+\alpha_{n}\left(\gamma \phi\left(x_{n}\right)-F \hat{z}\right) .
$$

Apply Lemma 2.9 to get

$$
\begin{aligned}
\left\|x_{n+1}-\hat{z}\right\|^{2} \leq & \left(1-\alpha_{n} \bar{\gamma}\right)^{2}\left\|x_{n}-\hat{z}\right\|^{2}+2 \alpha_{n}\left\langle\gamma \phi\left(x_{n}\right)-F \hat{z}, J\left(x_{n+1}-\hat{z}\right)\right\rangle \\
\leq & \left(1-\alpha_{n} \bar{\gamma}\right)^{2}\left\|x_{n}-\hat{z}\right\|^{2}+2 \alpha_{n}\left\langle\gamma \phi\left(x_{n}\right)-\gamma \phi(\hat{z}), J\left(x_{n+1}-\hat{z}\right)\right\rangle \\
& +2 \alpha_{n}\left\langle\gamma \phi(\hat{z})-F \hat{z}, J\left(x_{n+1}-\hat{z}\right)\right\rangle \\
\leq & \left(1-\alpha_{n} \bar{\gamma}\right)^{2}\left\|x_{n}-\hat{z}\right\|^{2}+\alpha_{n} \gamma\left(\left\|x_{n}-\hat{z}\right\|^{2}+\left\|x_{n+1}-\hat{z}\right\|^{2}\right) \\
& +2 \alpha_{n}\left\langle\gamma \phi(\hat{z})-F \hat{z}, J\left(x_{n+1}-\hat{z}\right)\right\rangle
\end{aligned}
$$

It follows that

$$
\begin{aligned}
\left\|x_{n+1}-\hat{z}\right\|^{2} \leq & \frac{\left(1-\alpha_{n} \bar{\gamma}\right)^{2}+\alpha_{n} \gamma}{1-\alpha_{n} \gamma}\left\|x_{n}-\hat{z}\right\|^{2}+\frac{2 \alpha_{n}}{1-\alpha_{n} \gamma}\left\langle\gamma \phi(\hat{z})-F \hat{z}_{J} J\left(x_{n+1}-\hat{z}\right)\right\rangle \\
\leq & \left(1-\frac{2 \alpha_{n}(\bar{\gamma}-\gamma)}{1-\alpha_{n} \gamma}\right)\left\|x_{n}-\hat{z}\right\|^{2}+\frac{2 \alpha_{n}(\bar{\gamma}-\gamma)}{1-\alpha_{n} \gamma}\left[\frac{1}{\bar{\gamma}-\gamma}\left\langle\gamma \phi(\hat{z})-F \hat{z}_{,} J\left(x_{n+1}-\hat{z}\right)\right\rangle\right. \\
& \left.+\frac{\alpha_{n} \bar{\gamma}^{2}}{2(\bar{\gamma}-\gamma)} M_{1}\right],
\end{aligned}
$$

where $M_{1}=\sup _{n \geq 1}\left\|x_{n}-\hat{z}\right\|^{2}$. By Lemma 2.2 and (3.16), we see that $x_{n} \rightarrow \hat{z}$. 
Remark 3.4. If $\gamma=1, F$ is the identity operator and $\varphi\left(x_{n}\right)=u$ in our results, we can obtain Theorems 3.1, 4.1, 4.2, 4.4 and Lemma 4.3 of Hong-Kun Xu [5].

Authors' contributions

The main idea of this paper is proposed by Meng Wen. All authors read and approved the final manuscript.

\section{Competing interests}

The authors declare that they have no competing interests.

Received: 28 November 2011 Accepted: 15 June 2012 Published: 15 June 2012

References

1. Banach, S: Surles opérations dans les ensembles abstraits et leur application aux equations intégrales. Fund Math. 3, 133-181 (1922)

2. Meir, A, Keeler, E: A theorem on contraction mappings. J Math Anal Appl. 28, 326-329 (1969). doi:10.1016/0022-247X (69) $90031-6$

3. Goebel, K, Reich, S: Uniform Convexity. In: Hyperbolic Geometry and Nonexpansive Mappings. Marcel Dekker, New York (1984)

4. Browder, FE: Convergence of approximations to points of non-expansive maps in Banach spaces. Arch Ration Mech Anal. 24, 82-90 (1967)

5. $\mathrm{Xu}, \mathrm{HK}$ : Strong convergence of an iterative method for nonexpansive and accretive operators. J Math Anal Appl. 314 631-643 (2006). doi:10.1016/j.jmaa.2005.04.082

6. Liu, LS: Iterative processes with errors for nonlinear strongly accretive mappings in Banach spaces. J Math Anal Appl. 194, 114-125 (1995). doi:10.1006/jmaa.1995.1289

7. Xu, HK: Iterative algorithms for nonlinear operators. J Lond Math Sov. 66, 240-256 (2002). doi:10.1112/ S0024610702003332

8. Bruck, RE, Passty, GB: Almost convergence of the infinite product of resolvents in Banach spaces. Nonlinear Anal. 3 , 279-282 (1979). doi:10.1016/0362-546X(79)90083-X

9. Bruck, RE, Reich, S: Nonexpansive projections and resolvents in Banach spaces. Houst J Math. 3, $459-470$ (1977)

10. Cai, G, Hu, CS: Strong convergence theorems of a general iterative process for a finite family of $\lambda_{i}$-strict pseudocontractions in q-uniformly smooth Banach spaces. Comput Math Appl. 59(1), 149-160 (2010). doi:10.1016/j. camwa.2009.07.068

11. Suzuki, T: Moudafi's viscosity approximations with Meir-Keeler contractions. J Math Anal Appl. 325(1), 342-352 (2007). doi:10.1016/j.jmaa.2006.01.080

12. Marino, G, Xu, HK: Convergence of generalized proximal point algorithms. Commun Pure Appl Anal. 3, $791-808$ (2004)

13. Jung, JS: Iterative approaches to common fixed points of nonexpansive mappings in Banach spaces. J Math Anal Appl. 302(2), 509-520 (2005). doi:10.1016/j.jmaa.2004.08.022

doi:10.1186/1687-1812-2012-98

Cite this article as: Wen and Hu: Strong convergence of an new iterative method for a zero of accretive operator and nonexpansive mapping. Fixed Point Theory and Applications 2012 2012:98.

\section{Submit your manuscript to a SpringerOpen ${ }^{\circ}$ journal and benefit from:}

Convenient online submission

Rigorous peer review

- Immediate publication on acceptance

- Open access: articles freely available online

- High visibility within the field

- Retaining the copyright to your article

Submit your next manuscript at $>$ springeropen.com 